\title{
An Innovative Evolution on 3D Multimedia Technology
}

\author{
Mohd Muntjir \\ Department of Information Technology, \\ College of Computers and Information Technology \\ Taif university Taif ,Saudi Arabia
}

\author{
Hussain Abu Sorrah \\ Department of Information Technology \\ College of Computers and Information Technology \\ Taif University Taif Saudi Arabia
}

\begin{abstract}
$3 \mathrm{D}$ multimedia provides an exclusive mix of multimedia and communication design process, design of $3 \mathrm{D}$ animation media and virtual reality, and 3D media connected with real and virtual studio and on-location multimedia evolution. It not only focuses on the practice and theory of the technical tools and techniques required for creating $3 \mathrm{D}$, but also on the creative art and design aspects of digital media, collective real and virtual reality media through the creation of design and technology skills, understanding and knowledge. The introductory International Workshop on Hot Topics in 3D multimedia was held in Singapore, in July 2010, in association with the IEEE International Conference on Multimedia. This Conference was sponsored by four different IEEE societies; International Conference on Multimedia and Expo is the IEEE flagship conference on Multimedia Technology. Hot3D was an animated workshop participated, with a remarkable compilation of papers screening different aspects of 3D multimedia Technique. Therefore, this report assembles some of the consequences offered at the workshop that will be more effective in future.
\end{abstract}

\section{Keywords:}

Multimedia, 3D, Fusion, Haptic

\section{INTRODUCTION}

With the advancement in communication, sensing, and visualization technology, 3D learning has become progressively integrated into real-world applications, from entertainment, architecture, and manufacturing to protection and preservation. The basic needs of this 3D technology are the evaluation of scene depth information, rather in real time. Areas such as computer graphics, robotics, and computer vision, have considered the mining of 3D information for more than 30 years, although it relics a demanding setback. Multimedia researchers must acquire the purity of depth information and other multisensory information into deliberation when manipulating their system, creating it an exclusive research prospect. Although, there already has been designated about the latest research consequences in the areas of modeling, conception, administration and performance of collaborating 3D multimedia content. Assessment and investigation in $3 \mathrm{D}$ multimedia is at a feasibly subversive status. The central robust compulsion of this uprising is $3 \mathrm{D}$ display technology. Henceforth, with the help of current technology improvement in that obverse, 3D displays are at the exciting of becoming widespread and reasonably rates and that may integrate even auto stereoscopic demos. On other side from distinct oversight, development in computational power -along with prevailing GPUs - has allowed an everincreasing verity in $3 \mathrm{D}$ display establishment. In current multimedia technology, 3D audio now always steadfastly integrated with 3D identifications, involving 3D sound field broadcast. Haptic systems are also being firmly incorporated within 3D systems. Furthermore, brand new depth scrutiny cameras, as well as brand new 3Dexploration and also activity algorithms is truly near to badge marketing-quality three dimensional object interpreting linked with authentic presentations, as opposed to being accessible to man-made displays as earlier. These variables mutually shape the "Perfect Storm": a place susceptible an excessive ignition market related with associated technology as well as platforms, through a pace connected with development.it does not easily fit in your cycle allied with classic conference discussions as well as journals. Relatively easy, however accurate locations with respect to expressive exploration from pioneering stages will be abundant, the certain animations Multimedia system community was without a proper place with respect to having reactions during establishment as well as primary stages from the improvement associated with fundamental as well as probable demanding expertise. This actually is the real void that Hot3D efforts for you to comprehensive, contribution a place affecting to animated discourse associated with early-stage, feasibly difficult consideration. Moreover, the occasion captivated a lot of researcher and investigators; working regarding selected subject areas concerning animations multimedia architecture. It brings out a distinct chance to attach to added researchers taking care of three dimensional Multimedia technologies, below a place made to complete conversation and also proposals within early on stageexploration, in addition to furnace totally innovative collaboration. Hence, such a report encapsulates this study accessible on the discussion.

\section{3D MULTIMEDIA ATTRIBUTES WITH CONTENTS}

With the expansion of computer techniques, 3D model has been used more extensively and content-based 3D model improvement has been a hotspot in the area of multimedia information recovery. How to extort 3D models' attribute inefficiently is still a complexity. Projection based 3D model attribute mining is a major kind in feature extraction, as of its heftiness against simplification, noise, and its effortlessness of feature extraction. Conceptual development in computing performance, along with expansion of platform autonomous 3D content values, opens the method for application of interactive $3 \mathrm{D}$ technologies in a diversity of domains such as tourism, education, cultural heritage, training, and ecommerce. Though, the prospective of $3 \mathrm{D}$ technology in daily applications can be completely subjugated only if complemented. We know about the haptically enhanced multimedia broadcasting organization. In order to illustrate convenience of the haptic system, some potential scenarios with haptic communication are scheduled. These scenarios can be classified into passive and active haptic communication scenario, which can be completely authored by scenario writers. At last, in order to demonstrate how the haptically improved scenario works, a distinctive example can be demonstrated to clarify exclusively for a home shopping setting. The important communication; Next Generation 3D Video Representation, Processing and coding was accessible from Disney Research. It statednumerousphases, from your current express related with animations articles invention, by technologies belowinnovation, to be able to evaluation constraints related with technologies, and studythough had to 
methodicallyoperate the specificskillsconnected with animations customer equipment in the future. Genuinelycontrolling area, a situation statement [3] was revealed through communicating the prominencerelating to extra analysis after 3D images Haptic. Yet, additionalcontext document [1] have been disclosed by just L Goldman, speaking around ones necessitate as well as situationassociated with $3 \mathrm{D}$ images high quality assessment.

\section{INTENSITY ESTIMATION AND 3D PLANNING IN MULTIMEDIA SYSTEM}

An enticing 3D inspecting skill is assembled on a attentive sight design of the content. Pigment is a extraordinary aspect that impacts human depth sensitivity. Former works in color and depth perception have shown that long-wavelength colors perform closer to viewers when contending with shortwavelength colors. D.Gandolf [2] scheduled any selfassurance place, which in turn blends the specific consistency along with quality of the match-up. Moreover, Itvisibly types this constancy of each alternative inference. Such an assurance map signifies esteemed, further information that can be leveraged in consequent steps of the $3 \mathrm{D}$ processing sequence. In this context, this paper offering an addition of a cross-bilateral filter that controls this consistency information during a fast-converging improvement step in order to create vigorous and dependable inequality plans. Yun Suk Kang [6] advocates this high-quality multi-view fascinating depth age group procedure using a number of complexions and in addition stimulating depth cameras. Just afterward-grasping low-resolution stimulating depth roadmaps by a few TOF video cameras, the specific stimulating depth data will be deformed in to shade impression parts in addition to employed since the primary variance appeal. By making use of the specific stereo system associated to employing observation broadcast while using the primary difference facts, this individual $\mathrm{s}$ obtain far more accurate in addition to secure multi-view variance roadmaps, while rivaled to persons' conclusions with no initial difference statistics. Furthermore, in shape from focus (SFF), brightness sounds deviation in addition to compelled functions mold the individual efficacy considering deliberate gauge operator video camera. Mendaparaet.al. Improve the engagement of complex wavelets caused by shift-invariance in addition to directionality on the variation for better model for revealing a variation of appearances, which regularly plays some sort of vital situation thorough valuation of any kind of pictures. [4]. Furthermore, a quadrature pair of steerable filters is involved to extent focus by estimating the local supported energy of the revealed configurations. Tentative models are offered to determine the efficiency of the method and the consequences equate productively to well-documented measures in fiction. Several 3D formats materialize and will co-exist for a long time since there is no 3D normal that defines a mostly established 3D format. Hence, the sustenance for several 3D formats will be substantial for alluring 3D into home. In [5] Zhang recommends a fast and functioning approach to distinguish whether an image is a $2 \mathrm{D}$ image or a $3 \mathrm{D}$ image determined with a couple of stereo images, and to further acknowledge the exact 3D format in the latter illustration. The technique calculates a control map; followed from image variances between the left and right view images; techniques the measurements from the distribution of edge widths combined with configuration alikeness analysis to perceive the manifestation of a 3D format and to classify the structure.

\section{3D MANIFESTATIONS AND REPRESENTATION WITH 3D SOUNDS}

A computer constructed multimedia material deals distinctive means of supportive 3D information illustrations. Viewing dynamic and 3D animations is assumed to be a possible way of changing and improving the incomplete mental models. However, based on several researchers, it is generate that $3 \mathrm{D}$ models may lead to perceptive overload efforts in hypermedia-learning surroundings in individual; as such atmospheres are expected to produce a substantial cognitive capacity. On the other hand, the findings of Ferk et al. (2003) research revealed that some representations of 3D structure are better assumed and can be more voluntarily used in solving tasks of different complexity. In Modern days, 3-D defines an image that specifies the sensitivity of depth. While 3-D images are made collaborating so that users sense involved with the panorama, the involvement is called virtual reality. We generally need a special plug-in viewer for our Web browser to view and interact with 3-D images. Virtual reality experiences may also require additional equipment. Standard commodities for creating 3-D effects comprise Light wave 3D, Extreme 3D, Studio MAX 3D, Ray Dream Studio, Visual Reality and Softimage 3D. Plenoptic camcorders are able to obtaining multi-view sizes that may be exposed about auto-stereoscopic reports. Depth maps can be reproduced from the set of plentiful visions. Though it is an introverted contact lens arrangement, mostly the question looks whether or not it is proper with regard to $3 \mathrm{D}$ as well as identical dimensions. The precise essential idea can be that the detail using which often with the ability to construct depth maps is static because of the aperture size on the key contact lenses. In [6] Drazic realizes the depth observation competences of plenoptic cameras. Moreover, an actual formula reckoning the specific aspect decision will exist in addition to reassuring to push the principal design and style collections for an exceptional detail controlling introverted lens programs. Multi-user auto stereoscopic presents have been founded within the European Union-funded MUTED and HELIUM3D progresses. These operate head rushing in order to express images that are accessible in sections specified to as exit pupils that follow the users' eye positions. In the MUTED, established images are formed on a direct-view liquid crystal display (LCD) with novel optics structured by the head tracker interchanging the straight backlights. Surmanet. al[9] explains the design and structure of the shows along with estimate consequences and future expansions, defining value of process, current status, and the multimodal latent of the HELIUM3D arrangement. Traditional 3D audio systems consistently have a controlled sweet spot for the user to characterize 3D effects marvelously. In [8] Song et. al.present a particular 3D audio system with loudspeakers that has unrestrained sweet spots. Hence, taking that methodology is usually to sustain a video camera track the authentic users brain actions, in addition to recomputed the real crosstalk canceller filter systems accordingly. The system would be the very first non-intrusive animations audio system of which familiarizes for you to both brain surroundings in addition to preference having 6-8 examples of objectivity. This appliance proposals in the future also been continued to add in room modeling [12] further enlightening the outcomes [13]. Hence, we can demonstrate about the 3D Images and 3D sound in modern days. 


\section{DESCRIPTIONS ABOUT THE SYNTHESIS AND VIDEO RESOLUTIONS IN 3D MULTIMEDIA}

The Research and Investigation on error flexibility in multiview coding is currently obtaining meaningful consequences. Although there is a congregation of literature regarding error recovery in $2 \mathrm{D}$ video, due to the arithmetical difference in motion compensation among temporal frames and inconsistency advantage among perspectives, such processes are inadequate to deliver to the inevitabilities of multi-view video recording. In [10], Dissanayake et. al. reports the above concern by broadcast of terminated inequity vectors for error recovery purpose. The method, which is related with all the Joint Scalable Movie Model (JSVM) codec along with particular by using simulated Internet Protocol (IP) box multilevel circumstances, can be effective along with a proper inaccuracy destruction system to dispense operative multiview video recommendation. The tentative results recommend that the planned algorithm immersions an irrelevant denial of quality in error free environments due to the existence of unnecessary chronicles. However, it regains the restructured picture quality meaningfully in error prone backgrounds, specifically for Packet Loss Rates (PLRs) greater than $7 \%$. Curiosity in 3D video conception systems is a never-emergent field. Such fields comprise the establishment of 3D content to users thus opening the consideration of $3 \mathrm{D}$ video statement and broadcast. To address communication and transmission one must consider error compliance. A Multiple Description Coding (MDC) can provide an enthusiastic video announcement over wireless networks. Even though, it could possibly familiarize with considerable degrees of in attention. In [11], Adedoyinet. al. recommend accessible MDC design using motion vector (MV) encoding for $3 \mathrm{D}$ videos. Tentative concerns explain that the algorithm can progress the frame feature by up to $2 \mathrm{~dB}$ over a pixel based expression scheme with exceptional coding while meaningfully reducing the bit rate attached to a pixel and motion interruption arrangements. In the multi-view video plus depth (MVD) demonstration for $3 \mathrm{D}$ video, a depth map sequence is coded for each sight. Henceforth, in the decoding end, a view synthesis algorithm is used to produce effective assessments from depth map plans. Many recognized view at functionality algorithms exposes demonstration objects exclusively on subject constraints. In [16], Chen et expresses a latest depth-level-adaptive appearance at functionality criteria that will lessens the number of artifacts in addition to improves the products this integrated image. The authentic algorithm presents understanding the interesting depth level so that no pixel worth in the integrated picture comes from pixels greater than one interesting depth level. Advancements with strive good quality in the constructed sights usually are noticed in just a several via being unfaithful test out situations, despite the fact that the actual particular good quality in the contributed strategy have been just like or maybe much better than that may in the checkout features strategy utilized by Relocating Picture Specialists Team (MPEG).Luat [14] Reveals his constant research in synthesis of free-viewpoint $3 \mathrm{~d}$ images with multi-view online video media with regard to 3DTV. Using the emergent breakthrough related with stereoscopic 3DTV, they have prolonged a latest reference free-viewpoint render algorithm to create stereoscopic views and scenes. Two identical options for increasing free-viewpoint animations multi-view online video media into a stereoscopic vision have been proposed. These types of selections take into account the intricacy in the algorithms through applying this redundancy inside music photos, given that they intention in a real-time components improvement. Regularly remedies arise from using a new horizontally transfer rather then two times setup in the reference point to free-viewpoint render algorithm for stereo creation, in order that the render time period is generally diminished through up to $30-40 \%$.Although the deal is that the rendering quality is $0.50 .9 \mathrm{~dB}$ lower than when applying FVP stereo conception. Concerns display that stereoscopic landscapes is generally produced by $3 \mathrm{~d}$ multiview video clip by making use of distinctive advantages within stereoscopic backgrounds, comprising the exact positioning, characteristics within textures as well as insignificant instructions.

\section{ADDITIONALCONTEMPORARYINN OVATIONS IN 3D MULTIMEDIA TECHNOLOGY}

Modern improvements in 3D technologies and measurement instrumentation collective with multimedia databases offer today innovative options for the incorporated and comprehensive explanation of Cultural Heritage entities.3D multimedia is exceptionally active on these days. In ICME at Singapore, regardless from the Hot3D workshop, there was a board about 3D multimedia; in addition to several additional procedures acknowledged features coupled with 3D.The board, entitled "3D Multimedia: Research Status and Opportunities" was controlled by Dinei Florencio, from Microsoft Research, and appreciated with a number of expert in the research areas. These involved, AljoschaSmolic, from Disney Research, Phil Surman, from De Montfort University, Eckehard Steinbach, from TU. Furthermore, many papers from the conference were coverage applicable outcomes on 3D multimedia. Trocanet al, [15] considers the use of compressed sensing in multi-view imaging, suggesting an algorithm that hustles image recovery using the projectiondomain residual between the random dimensions of the image in question and a disparity-based prediction generated from attached, high-quality images. One of the papers in 3D audio won one of the ICME best paper awards [19], and has lately been prolonged to appraise parameter compassion [17]. It improves the accuracy of 3D sound source localization (SSL) by up to one order of importance compared to the state of the technique [18], by using the advance evidence accessible on early reproductions. It outlines on a scarcity-motivated room shaping procedure [10] and on current significances onmaximum prospect SSL [20]. Additionally, another exciting paper on 3D sound [21] places sounds on a 3D virtual background for user navigation through a panorama. Furthermore, one of the imperative applications of 3D is the area of remote connection. That area has some current refreshing conclusions relating to pseudo-3D [22] with a fascinating paper from HP labs [23] appraising eye interaction. Hence, Intellectual Expansion of 3D interactive network applications needs standards for expressive 3D content as well as metadata principles for recitation the content.

\section{FUTURE SCOPE}

In terms of perception, innovative work in $3 \mathrm{D}$ processing could comprise of studying functionalities jointly and not discretely.

3D Multimedia is an integration of gaming industry, education, mobile phones, PDAs, simulation, toys, film titling, commercials, special effects, web entertainment programs, broadcasting, etc.3D Multimedia is a creative industry for the creative people, based on one's aspiration, creative skills, experience $\&$ expertise. Research in 3D 
Multimedia technology is really developing at the massive hustle. Development in numerous fronts is been manageable, and indicative improvements are been accomplished in areas like 3D audio, 3D displays, 3D virtual atmospheres free viewpoint construction, 3D suggestion and many mores. We suppose this area to be very exciting for the next some years, accomplishing with a common 3D displays and environments. We have some stimulating years onward.

\section{REFERENCES}

[1] L.Goldman and T. Ebrahimi, "3d quality is more than just the sum of $2 d$ and depth," in Hot3D (ICME'2010), 2010.

[2] J.Jachalsky, M. Schlosser, and D. Gandolph, "Confidence evaluation for robust, fast-converging disparity map refinement," in Hot3D (ICME'2010), 2010.

[3] E. Steinbach, R. Chaudhari, and J. Kammerl,"Current status and future directions of haptics in $3 \mathrm{~d}$ multimedia," in Hot3D (ICME'2010), 2010

[4] P. Mendapara, A. Baradarani, and Q. M. J. Wu, "An efficient depth map estimation technique using complex wavelets," in Hot3D (ICME'2010), 2010

[5] T. Zhang, "3d image format identification by image difference," in Hot3D (ICME'2010), 2010.

[6] V. Drazic, "Optimal depth resolution in plenoptic imaging," in Hot3D (ICME'2010), 2010

[7] Y. S. H. Yun-Suk Kang, "High-quality multi-view depth generation using multiple color and depth cameras," in Hot3D (ICME’2010), 2010

[8] M.S. Song, C. Zhang, D. Florencio, and H. G.Kang, "Personal 3d audio system with loudspeakers," in Hot3D (ICME’2010), 2010

[9] P.Surman, R. Brar, I. Sexton, and K. Hopf, "Muted and helium3d auto stereoscopic displays," in Hot3D (ICME’2010), 2010

[10] M.B.Dissanayake, D. Silva, S. Worrall, and W. Fernando, "Error resilience technique for multi view coding using redundant disparity vectors," in Hot3D (ICME’2010), 2010.
[11] S. Adedoyin, W. Fernando, and A. Kondoz, "Scalable mdc for 3d stereoscopic video using motion vector encoding," in Hot3D (ICME'2010), 2010

[12] D. Ba, F. Ribeiro, C. Zhang, and D. Florncio, "L1 regularized room modeling with compact microphone arrays," in ICASSP, 2010

[13] M.S. Song, C. Zhang, D. Florencio, and H.G.Kang, "Enhancing loudspeaker-based 3d audio with room modeling," in MMSP, 2010

[14] P. H. N. D. W. Luat DO, Svitlana Zinger, "Conversion of free-viewpoint $3 \mathrm{~d}$ multi-view video for stereoscopic displays," in Hot3D (ICME'2010), 2010

[15] M. Trocan, T. Maugey, J. Fowler, and B. PesquetPopescu, "Disparitycompensated compressed-sensing reconstruction for multi-view images," in ICME, 2010

[16] Y. Chen, W. Wan, M. Hannuksela, J. Zhang, H. Li, and M. Gabbouj, "Depth-level-adaptive view synthesis for 3d video," in Hot3D (ICME’2010), 2010

[17] C. Zhang, D. Florencio, and Z. Zhang,"Why does phat work well in low noise reverberative environments?" in ICASSP, 2008

[18] F. Ribeiro, C. Zhang, D. Florncio, and D. Ba, "Using reverberation to improve range and elevation discrimination for small array sound source localization," IEEE Trans. on Audio, Speech and Lang. Proc., vol. 18, no. 7, pp. 1781-1792, Sept 2010

[19] F. Ribeiro, D. Ba, C. Zhang, and D. Florncio, "Turning enemies into friends: Using reflections to improve sound source localization," in ICME, 2010

[20] C. Zhang, Z. Zhang, and D. Florencio, "Maximum likelihood sound ' source localization for multiple directional microphones," in ICASSP, 2007

[21] W. Ma, Y. Liu, Y. Wang, Y. Xu, H. Zha, and W. Gao, "Interactive viewpoint-space navigation for visual-audio exhibition of painting," in ICME, 2010

[22] C. Zhang, Z. Yin, and D. Florencio, "Improving depth perception with " motion parallax and its application in teleconferencing," in MMSP, 2009

[23] D. Florencio and C. Zhang,"Multi-view video compression and streaming based on predicted viewer position," in ICASSP, 2009 